\title{
The Analysis and Application Research of the System Structure Model for Software Engineering
}

\author{
WANG Zhonghua ${ }^{1, \text { a }}$ \\ ${ }^{1}$ School of Electronic Information Engineering, QiongZhou University, Sanya City 572022,China \\ aWANGZhonghua1028@yeah.net
}

Keywords: System Structure Model; Software Engineering k; MDA

\begin{abstract}
With the development of human society and scientific technology, people's material production and social activities, cultural activities, and increasing the scale of scientific research, social contact is increasingly close, between departments of human's epistemological and methodological level is increasing day by day, gradually from the height of the overall and the system to recognize and solve the problem, to form the concept of system theory and related theoretical system. This article is around the software engineering of the defects in the system structure model conversion technology. On the basis of the focus on design pattern oriented model transformation method, the model of knitting and the metadata mapping were studied, based on the model driven architecture (MDA) is designed for Web application development platform, verify the validity of the above technologies and methods.
\end{abstract}

\section{Introduction}

With the rapid development of information technology, especially the prosperity of the Internet has greatly changed the lifestyle of human society. A lot on the Internet as the carrier of the software system appear constantly, such as electronic commerce, electronic government affairs, battle command systems and network game network software is applied to the economy, society, military, entertainment and other fields, to the progress of human society played an immeasurable role. These systems instead of a single technology, relatively closed, compared the static structure of the software system, presents the heterogeneity, the characteristics of the open and dynamic [1].

The thought of software engineering, is in the early 1980s by Dr. Winston W. Royce put forward for the first time. He believes that the lack of system engineering method of the project, is likely to lead to software cannot be run on the selected hardware, software can't with other software and hardware system integration, software is not suitable for use for a long time [2]. In product development, oversight system, long-term not solve the software crisis will intensify. This article from the Angle of the engineering system theory to the software engineering investigation and analysis, the structure model of the elements and the mutual relationship are analyzed. Use of the established software engineering system structure model, this paper discusses the general steps in the process of software engineering, the key of object-oriented design pattern model transformation method, the model of knitting and the metadata mapping were studied, based on the model driven architecture (MDA) design for Web application development platform, verify the validity of the above technologies and methods.

\section{Overview of the software engineering related technology}

Related with the development of science and technology, computer technology has also obtained fast development, accordingly software quantity more and more, the scale of software is more and more big, the individual characteristics of many programs makes software more and more difficult to maintain, and produces the software crisis. In order to solve software crisis, from two aspects of technical measures and necessary organization management measures research how to better development and maintenance of computer software, and then the software engineering this emerging subject. The concept of software engineering system is to examine some of the thoughts of system theory, application engineering and research on software engineering science system, 
according to the general principle and method of system theory, system theory and engineering analysis software engineering system and the basic rules of inner character [3]. Software engineering systems as a complex system, in order to develop software products meeting customer requirements and formed a complete system, the internal elements of system coordination, division of labor cooperation, strive for the prescribed under the requirement of cost and schedule, to efficiently complete the established goals. The basic elements of the software product demand, operation, developer divide, use and supervision party, problem, demand, software, support technology and development tools.

Model driven architecture (MDA), the purpose is to improve the efficiency of software development, it and the component oriented and service oriented, and the software architecture based on the distribution of the middleware software development environment complement each other, has become a current in a heterogeneous environment is an effective means of developing large-scale software, is one of the hot research topic in the field of software engineering. Model driven architecture research centered on the model of software development methods, compared to traditional code-centric software development method is closer to the application, characteristics such as higher abstract level, usually by its development of software can run in a heterogeneous platform support, can be effectively applied to develop all kinds of large-scale complicated information system based on network [4]. Briefly the process of MDA method is shown in figure 1.

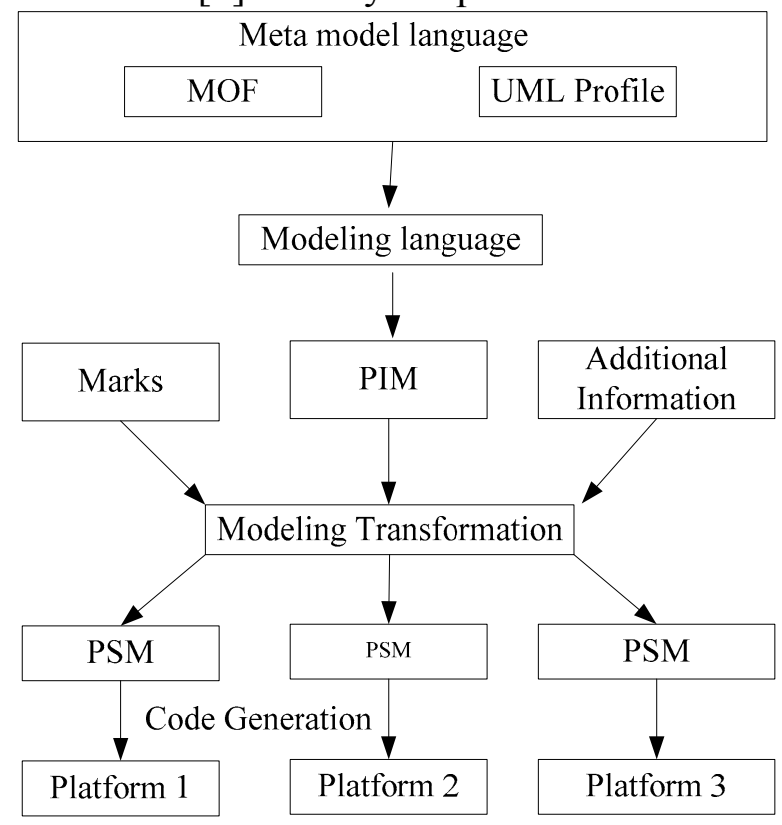

Figure 1. The process of MDA method

\section{Software engineering process analysis of the software engineering system}

In software engineering system, from the global, the perspective of the overall grasp of software engineering. Mind mapping in system theory can better describe the process of software engineering [5]. Combined with the structure of the software engineering system model, it can put the software engineering software engineering process in the system diagram in figure 2. 


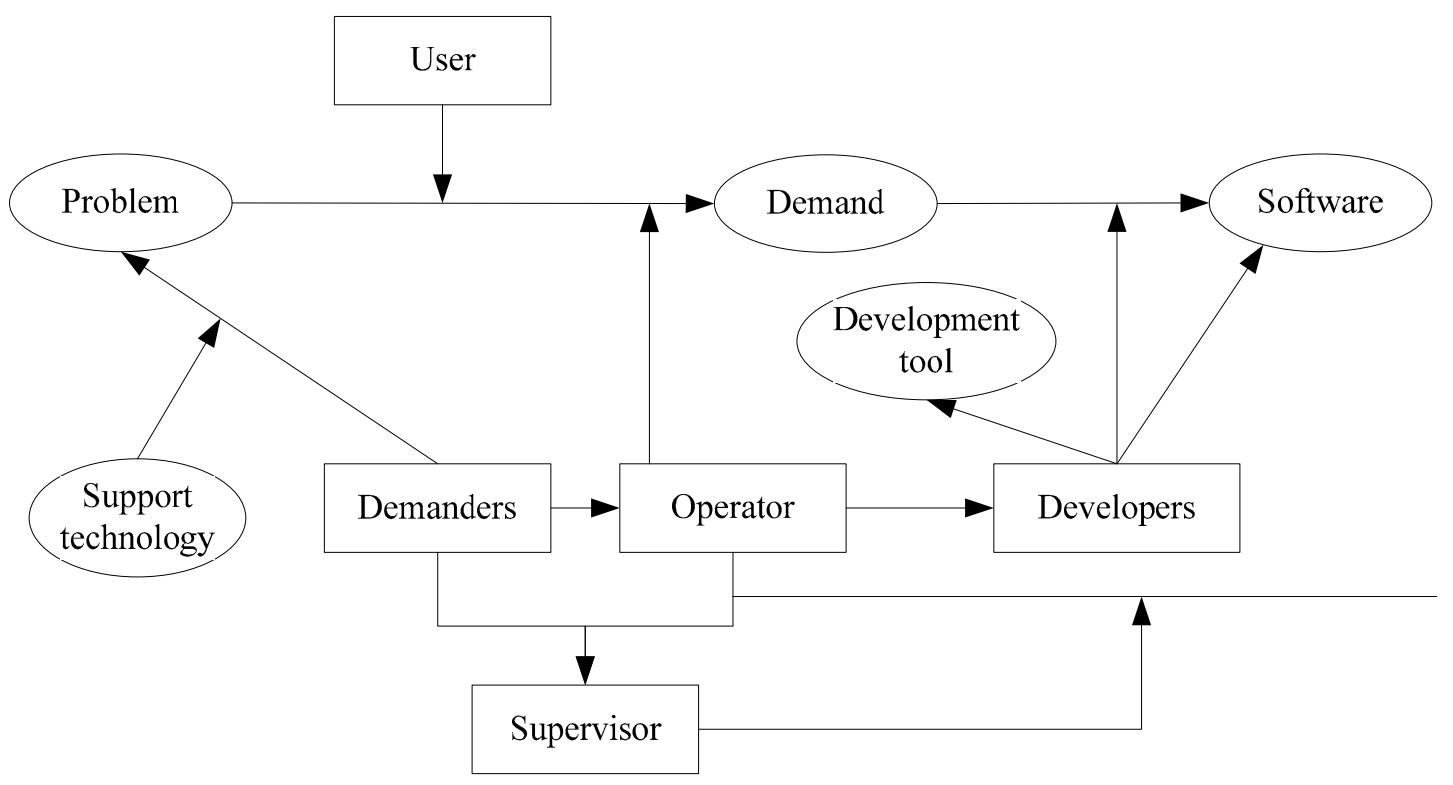

Figure 2. Software engineering process of the software engineering system

From the software engineering process of mind mapping, you can see that in the process of software engineering, there are two main line. A starting is from the problem elements, elements into demand, again into the software process. A requirement, choose the corresponding operation, operation side developer divide, developer divide development to the software elements. The two lines is also important to software engineering process. From the elements in the process of problem elements into requirements, pay attention to the corresponding demand and operates the description of the problem is consistent, if the difference is bigger, and is not found in time, is likely to lead to the failure of the software project or increase the workload of modified demand. Transformation process from requirements to software elements, is done by developer divide development activities, therefore, the developer divide is also the key.

\section{Web application development platform design based on the MDA}

Web application is an important platform, the Internet information communication software enterprises to undertake a large Web application design, development, debugging and maintenance of heavy work, code-centric gradually to meet the demand of the existing software development technology. To solve the above problems, this chapter was designed and implemented based on the model of knitting and the metadata mapping technology of Web application development platform. Except has all kinds of Web application oriented modeling tools and model warehouse, the code template library such as the underlying supporting facilities, also has a dynamic alternative model of converter and the code generator. Users describe business models can be automatically generated Web application prototype system and the back-end database. Web application development platform based on the MDA structure is shown in figure 3. System is divided into four layers, system layer, respectively, the metadata layer, data layer and the underlying infrastructure.

System layer provides system testing to start, graphical interface, as well as the function of the external Web application server interface, realize integrated Web development, operation and debugging environment.

Metadata layer definition base model, development framework meta-model, weaving model and model extension interface converter. Metadata layer provides a dynamic alternative model converter mechanism. It to model defines the weaving methods from base model into the development model transformation rules, and generate a data layer model of the converter.

The data layer provides Web application development platform based on MDA modeling tools, model converter, the code generator and a code generator extension interface. Its main function is to realize from the domain model to the target set of code generation mechanism. Warehouse 
infrastructure provides the model, model test, the model transformation engine, and the code template library and other infrastructure to support layer data modeling, transformation, and code generation capabilities.

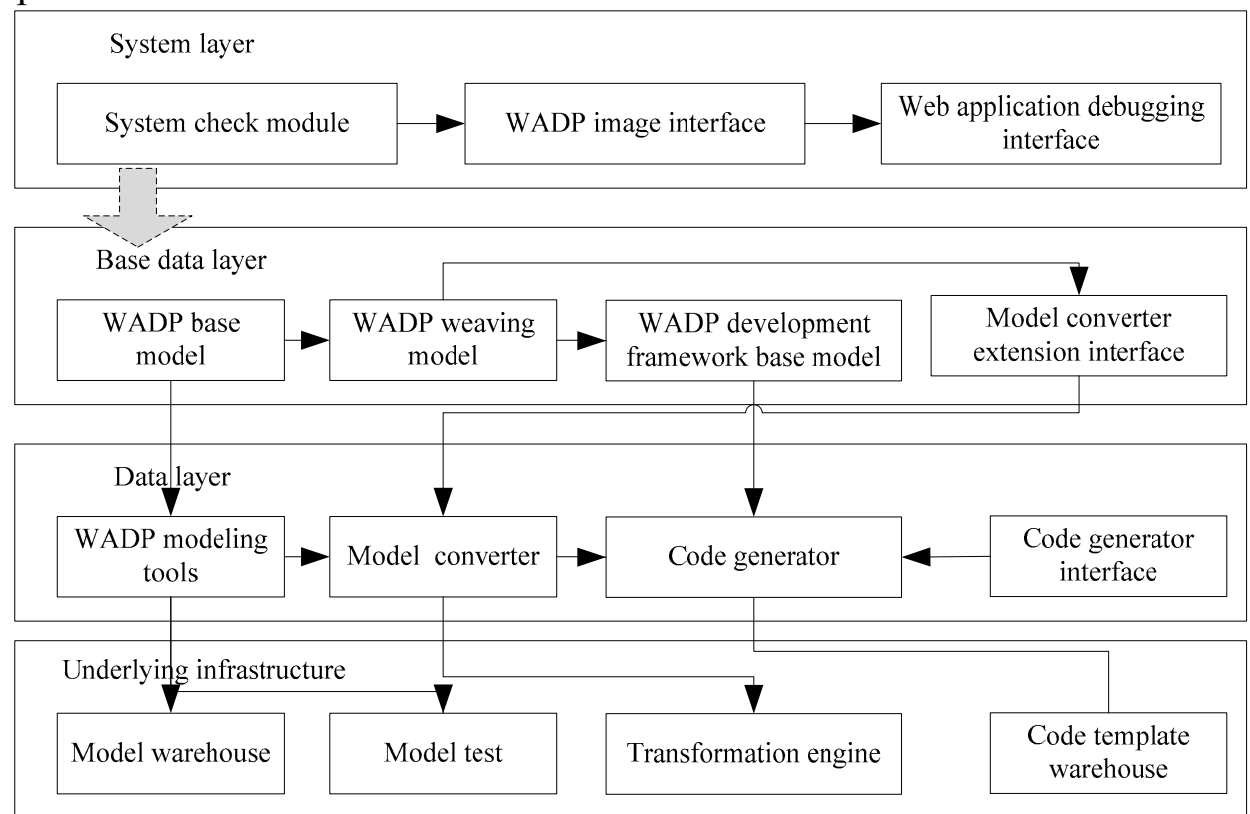

Figure 3. Structure of Web application development platform based on the MDA

\section{Conclusion}

The constant enlargement of the range of application and software system complexity continues to increase, caused the traditional code-centric software engineering development method is difficult to meet demand. Software engineering as an emerging discipline, its related knowledge! Theory and method has been developing rapidly, it has a gradually deepening the understanding of the process. For the concept of software engineering system and the understanding of the relevant contents will also with the deepening of the study of it, and to understand gradually, finally be used. This article is based on earth-sized in Web application software development for the application background, around the software engineering development process a number of deficiencies in a series of research, and provide related support platform.

\section{Acknowledgements}

The natural science foundation of Hainan Province(613170).

\section{Reference:}

[1] P. Runeson, M. Höst: Empirical software engineering, Vol.14 (2009) No.2, p.131.

[2] A. Clauset, C.R. Shalizi, M.E. Newman: SIAM review, Vol.51 (2009) No.46, p. 661.

[3] P. Runeson, M. Höst: Empirical software engineering, Vol.14 (2011) No.2, p. 131.

[4] B. Kitchenham, O. Pearl Brereton, D. Budgen: Information and software technology, Vol.51 (2010) No.1, p. 7.

[5] D.L. Moody: Software Engineering, Vol.35 (2011) No.6, p. 756. 